\title{
Biochemical adaptations of mammalian hibernation: exploring squirrels as a perspective model for naturally induced reversible insulin resistance
}

\author{
C.-W. Wu, K.K. Biggar and K.B. Storey \\ Department of Biology, Institute of Biochemistry, Carleton University, Ottawa, ON, Canada
}

\begin{abstract}
An important disease among human metabolic disorders is type 2 diabetes mellitus. This disorder involves multiple physiological defects that result from high blood glucose content and eventually lead to the onset of insulin resistance. The combination of insulin resistance, increased glucose production, and decreased insulin secretion creates a diabetic metabolic environment that leads to a lifetime of management. Appropriate models are critical for the success of research. As such, a unique model providing insight into the mechanisms of reversible insulin resistance is mammalian hibernation. Hibernators, such as ground squirrels and bats, are excellent examples of animals exhibiting reversible insulin resistance, for which a rapid increase in body weight is required prior to entry into dormancy. Hibernator studies have shown differential regulation of specific molecular pathways involved in reversible resistance to insulin. The present review focuses on this growing area of research and the molecular mechanisms that regulate glucose homeostasis, and explores the roles of the Akt signaling pathway during hibernation. Here, we propose a link between hibernation, a well-documented response to periods of environmental stress, and reversible insulin resistance, potentially facilitated by key alterations in the Akt signaling network, PPAR- $\gamma /$ PGC- $1 \alpha$ regulation, and non-coding RNA expression. Coincidentally, many of the same pathways are frequently found to be dysregulated during insulin resistance in human type 2 diabetes. Hence, the molecular networks that may regulate reversible insulin resistance in hibernating mammals represent a novel approach by providing insight into medical treatment of insulin resistance in humans.
\end{abstract}

Key words: Metabolic depression; T2DM; PPAR- $\gamma$; MicroRNA; Glucose transport; Akt

\section{Introduction}

Type 2 diabetes mellitus (T2DM) is one of the most common metabolic diseases in the world, affecting more than 21 million people in the United States (1). Although the molecular basis of the disease is very well studied, cures and preventions for the disease have not been discovered. The pathogenesis towards T2DM is a result of a combination of metabolic dysfunctions, primarily characterized by severe insulin resistance and dysfunctional pancreatic $\beta$-cells resulting in hyperglycemia (2). Development of T2DM is thought to be caused by a combination of dietary behaviors, physical fitness, and genetic factors (3). Initiation of T2DM development is facilitated by a disturbance of normal biological functions in numerous tissues, with each tissue exhibiting both common and specific modes of dysfunction. Critical metabolic defects include insulin resistance in skeletal muscle, increased glucose production in the liver, and a progressive decline in insulin production in the pancreas. The physiological results in each tissue are caused by cellular dysregulation at the molecular level since cellular signaling processes such as glucose transport, insulin signaling, and mitochondria $\beta$-oxidation of fatty acids have all been found to be differentially dysregulated in the majority of T2DM patients $(4,5)$. One of the most prevalent phenotype associations with T2DM is an increase in adipocyte diameter associated with obesity (6). Although

Correspondence: K.B. Storey, Department of Biology, Institute of Biochemistry, Carleton University, 1125 Colonel By Drive, Ottawa, ON, K1S 5B6, Canada. Fax: +613-520-3749. E-mail: kenneth_storey@carleton.ca

Presented at the XLI Annual Meeting of the Sociedade Brasileira de Bioquímica e Biologia Molecular, Foz do Iguaçu, PR, Brazil, May 19-22, 2012. 
not all diabetics are obese and not all obese individuals are diabetic, there is a strong association between the two pathological conditions. The increase in adipose content in obese individuals can cause a disruption in endocrine production, and this change in hormonal balance can lead to an altered metabolism and often contributes to insulin resistance (3).

Currently, most of the research on T2DM has been conducted using human cell lines and rat/mouse models, where the diabetic condition can be experimentally induced. However, naturally insulin-resistant models, such as the hibernating 13-lined ground squirrel (Ictidomys tridecemlineatus), may provide a critical vantage point towards the treatment of human T2DM. This mammal naturally undergoes a massive increase in body fat accumulation, while facilitating a reversible resistance to insulin during bouts of the hibernation cycle (7). Hibernation is an altered physiological state that is characterized by seasonal heterothermy, entry into long periods of torpor at low body temperature $(\mathrm{Tb})$ that are interspersed with short arousals back to a normal $\mathrm{Tb}$ $\left(\sim 37^{\circ} \mathrm{C}\right)$, and a switch to a primary dependence on lipid catabolism for metabolic fuel (8). In preparation for hibernation, ground squirrels undergo hyperphagia to increase their body fat storage via the accumulation of triglycerides in white adipose tissue (9). This intense feeding allows the animals to increase their body weight up to $\sim 40 \%$, facilitating survival for many months during the hibernation season (10). The rapid development of an obesity-like state in these animals is a result of naturally induced insulin resistance during hyperinsulinemia, as well as an increase in adipocyte diameter (7). Once adequate fat storage has been satisfied, the animal is able to enter hibernation. During deep torpor, the metabolic rate is strongly depressed and physiological actions, such as breathing and heartbeat, fall to low levels while Tb drops to near ambient (10). Although maximal insulin resistance takes place during pre-hibernation, it is hypothesized that the reversal of insulin resistance takes place during the initial months of hibernation. It should be noted that metabolic rate depression and entry into a hypometabolic state is not unique to mammalian hibernators. When confronted with an extreme environmental stress, many animals escape by depressing their metabolic rate (often by $70-99 \%$ ) and enter a state of hypometabolism, variously called torpor, dormancy, estivation, anaerobiosis, diapause, etc., depending on the environmental and physiological cues involved $(11,12)$.

The molecular mechanisms of metabolic rate depression include global suppression of energy-expensive cell functions (e.g., protein synthesis, gene transcription, ATP-dependent ion pumps), reprioritization of ATP use by vital cell functions, and enhanced expression of multiple preservation mechanisms (e.g., antioxidants, chaperones) that protect and stabilize cellular macromolecules (12). Although it might appear that only a few connections exist between hibernation and T2DM, many of the metabolic pathways regulating hibernation are also frequently found to be dysregulated in T2DM. However, these T2DM-like dysregulations are readily reversed in ground squirrels once they arouse from hibernation. The ability to reversibly induce insulin resistance to achieve an obesity-like state suggests a possible mechanism allowing hibernators to actively regulate their endocrine system, and modulate their lipid metabolism. More importantly, during hibernation, ground squirrels provide a unique model that readily reverses the insulin resistance seen in T2DM without detrimental consequences.

In this review, we first focus on the biochemical pathways that are frequently altered and contribute to T2DM onset with particular emphasis on the same pathways that may contribute to natural insulin resistance during hibernation. Interestingly, both ground squirrels in hibernation and T2DM patients share differential regulations in a number of identical metabolic pathways that include the Akt signaling pathway, glucose transport pathway, and peroxisome proliferator-activated receptor$\gamma($ PPAR- $\gamma$ )/PPAR- $\gamma$ coactivator 1-alpha (PGC-1 $\alpha$ ) signaling. However, ground squirrels appear to exhibit a different mode of regulation of these pathways during hibernation compared to T2DM patients, implicating the important roles of these pathways in achieving reversible insulin resistance. We aim to profile the three major metabolic pathways mentioned above, and compare their relative modes of regulation during reversible insulin resistance (hibernation), and non-reversible insulin resistance (T2DM). In addition to these models of metabolic signaling pathways, the involvement of a novel regulatory mechanism - non-coding RNA - in hibernation is also discussed along with the possible roles for non-coding RNA in contributing to the pathogenesis of T2DM metabolic dysregulation. Here, we introduce hibernation, a well-documented response to periods of environmental stress, and the potential of reversible insulin resistance using the ground squirrel as an important experimental model.

\section{Metabolic dysregulation of T2DM and hibernation metabolism}

The pathogenesis of T2DM begins with defects in the body's basic metabolism. Upon normal feeding, increased glucose levels are balanced through the release of insulin, promoting the uptake of glucose and glycogen synthesis (13). In normal energy metabolism, metabolic fuels (glycogen and triglycerides) are stored, acting as reserves to be broken down during the fasting state. Insulin is a key hormone in metabolic regulation, monitoring and maintaining the glucose balance within the body. However, the maintenance of whole body glucose metabolism involves the coordination of multiple metabolic processes in multiple tissues. Dysregulation of any of these metabolic 
processes can lead to a disruption in total body glucose homeostasis, followed by the onset of insulin insensitivity and T2DM (14). Previous studies have implicated defects in several cellular processes in the contribution to T2DM; these include the previously mentioned glucose transport pathway, PPAR- $\gamma /$ PGC- $1 \alpha$ signaling pathway, and the Akt signaling pathway $(4,5,15,16)$.

Buck et al. (17) have previously observed that ground squirrel serum insulin levels are elevated by $\sim 4$-fold during September-October (periods of hibernation preparation) compared to summer animals; serum insulin levels continue to be elevated ( $\sim 4.5$-fold) during the initial months of ground squirrel hibernation. A 2-fold elevation of basal insulin levels is commonly associated with insulin resistance in T2DM in human patients; this could suggest that a dramatic increase in ground squirrel serum insulin may be a result of insulin resistance in the preparatory stages for hibernation, as well as the early months of hibernation. However, serum insulin has been found to return to basal levels in ground squirrels during late hibernation (December-January), suggesting a reversibility of insulin resistance (17). This dramatic reduction of serum insulin levels during hibernation suggests that, although the maximal insulin level is observed before entry in hibernation, the molecular mechanisms that regulate the reversible insulin resistance take place during hibernation, when ground squirrels undergo major changes in physiological actions in order to achieve a state of hypometabolism. Interestingly, not unique to ground squirrels, other hibernating mammals also exhibit patterns of insulin resistance during hibernation. Hibernators such as yellow-bellied marmots showed significant increases in plasma insulin levels during periods of weight gain characterized with peripheral insulin resistance and hyperinsulinemia (18). In vitro studies also showed that glucose oxidation of adipose tissue upon insulin stimulation in hibernating dormice was drastically reduced compared to active dormice, suggesting insulin resistance during hibernation (19). Similar results were found in hibernating hedgehogs, where a lack of insulin response was found in adipocytes at low temperature (20). Altogether, the metabolic adaptation of hibernation appears to include not only the regulation of insulin levels, but also of tissue sensitivity to the hormone.

Hibernation is an altered physiological state that is characterized by the extreme depression of basal metabolism. This type of metabolic suppression is favorable for organisms inhabiting environments that are subject to drastic temperature changes, as well as fluctuations in food and water availability. A current main model for hibernation studies is the 13-lined ground squirrel, I. tridecemlineatus, a rodent that is widespread in North America. A typical annual cycle of these ground squirrels includes a long period of hibernation that averages about 240 days (21). The hibernation season is characterized by long periods of dormancy (up to
1-2 weeks) during which $\mathrm{Tb}$ falls to low values that are interspersed with brief arousals (about $24 \mathrm{~h}$ ) back to euthermia. Hibernation is characterized by an altered approach to cellular energy management. In order to achieve a suppression of $\sim 90 \%$ compared to basal metabolic rate in euthermia, the ground squirrel must also suppress thermogenesis to allow $\mathrm{Tb}$ to fall to near ambient by overriding the normal homeostatic controls on $\mathrm{Tb}$ that are used in euthermia. However, it must be noted that the depression of $\mathrm{Tb}$ is a result of suppression of metabolic activities, suggesting that the drop in $\mathrm{Tb}$ is a regulated process (22). In order to achieve and maintain a low energy status, selective changes in gene expression have to take place to induce or suppress selected metabolic processes (12). Another mechanism that has previously been shown to play a central role in creating a hypometabolic state is reversible protein phosphorylation (RPP) (12). Since the entry into and arousal from a torpor state is a short process and requires rapid changes in many metabolic processes, RPP has been shown to be a primary mechanism responsible for suppressing and activating a variety of metabolic functions (23). Overall, in order to effectively achieve hibernation, the animals must 1) increase their metabolic fuel reserve in preparation for hibernation, 2) switch to a primary dependence on the catabolism of stored lipid reserves for metabolic fuel, and 3) reversibly suppress all non-essential ATP consuming processes (12). The suppression of non-essential ATP consuming cellular activities includes a wide range of metabolic processes ranging from a reduction in gene transcription, protein translation, cell cycle division, active ion pumps, and carbohydrate metabolism (23-25).

\section{Obesity-like pathology: hibernation vs T2DM}

In order to survive over long winter months without eating, hibernators undergo obligatory hyperphagia to increase their adipose storage, which can later be utilized as metabolic fuel. During the process of hyperphagia, induction of insulin resistance assists the hibernators in developing an obese-like state, a condition that is often associated with T2DM. The onset of insulin resistance in hibernators is thought to be induced during the preparation phase of hibernation, a period of hyperinsulinemia ( $\sim$-fold increase in serum insulin) (17). It has also been proposed that a decrease in the levels of leptin is a primary factor that permits hyperphagia (26). Since leptin is an adipose-derived hormone, the strategic suppression of leptin to facilitate hyperphagia suggests that the altered adipose metabolism in ground squirrels is a coordinated process. Although leptin resistance might play a role in the onset of insulin insensitivity, the development of obesity in T2DM is often a result of defects in adipose metabolism, which results in an uncoordinated release of non-esterified fatty acids, 
adipose-derived hormones, and various cytokines (27). It is possible that the obesity-like state observed in both hibernation and T2DM is a result of hyperinsulinemia, or vice versa; however, the mechanisms utilized by the hibernators to actively regulate adipose metabolism during torpor may provide an understanding of the basis of reversible insulin resistance.

\section{Glucose transport in T2DM and hibernation}

Skeletal muscle is the largest insulin-sensitive organ, and acts as one of the primary tissues that are responsible for blood glucose transport (3). The primary mechanism of glucose transport in skeletal muscle mainly involves two isoforms of the glucose transporter protein: glucose transporter type 1 (GLUT-1) and GLUT type 4 (GLUT-4). The central facilitator in insulin-induced glucose transport is GLUT-4, which is responsible for transporting $\sim 80 \%$ of the total glucose content from the bloodstream into skeletal muscle $(4,28)$. Upon insulin stimulation, GLUT-4 translocates from intracellular pools to the plasma membrane, the site of glucose uptake (Figure 1A) (29). Upon glucose transport into myocytes, the glucose is phosphorylated by hexokinase to become glucose-6-phosphate, and is either immediately catabolized through glycolysis or stored as glycogen (30). Experimental studies have shown that Wistar rats (Rattus norvegicus), induced to show diabetic pathology through streptozotocin injection, exhibit a decrease in the expression of GLUT-4 proteins in both the white and red skeletal muscle when compared to non-diabetic controls (4). In a separate study utilizing the same experimental model, the decrease in GLUT-4 expression was also accompanied by a decrease in the $V_{\max }$ activity of the glucose transporter in the plasma membrane (29). Taken together, these findings suggest that glucose transport is defective due to a decrease in both the expression and activity of GLUT-4 in diabetic-induced rats. However, other studies have shown that the expression levels of GLUT-4 are relatively similar in diabetic and non-diabetic patients, suggesting that the impaired glucose transport activities are mainly due to a defect in the delivery of GLUT-4 to the plasma membrane (31). Nevertheless, evidence strongly suggests that an overall disruption in glucose transport processes can lead to the onset of hyperglycemia in T2DM, where the defects can be attributed to impaired GLUT-4 translocation (Figure 1B), a decrease in GLUT-4 transport activity, downregulation of GLUT-4 expression, or any combination of the three.

To date, there has been limited research on the glucose transport system in hibernating ground squirrels. Recently, a study of the roles of myocyte enhancer factor-2 (MEF-2) transcription factor in myogenesis in skeletal muscle of hibernators found that there was an increase in the levels of the active MEF-2 isoform during late torpor (32). MEF-2 regulates a wide range of muscle-related cellular processes such as muscle growth and differentiation (33). More importantly, MEF-2 can regulate muscle glucose homeostasis by enhancing the expression of GLUT-4 via interactions with the GLUT-4 gene promoter (34). Experiments using transgenic mice have found that MEF-2 DNA binding activity was significantly reduced in insulin-deficient diabetic mice; however, the binding activity was rescued upon insulin treatment (34). This suggests that MEF-2 activity is strongly associated with insulin levels, and plays a critical role in modulating glucose balance. During late hibernation of ground squirrels, an increase in MEF-2 protein expression and DNA-binding activity was correlated with an increase in GLUT-4 expression, suggesting that glucose transport might be enhanced during hibernation (Figure 1C). However, previous studies in T2DM patients have shown that a decrease in glucose transport capacity was associated with a defect in the translocation of GLUT4 from the intracellular pool to the plasma membrane, rather than the changes in the expression of GLUT-4 (31). Nevertheless, an increase in GLUT-4 expression through MEF-2 activation during hibernation might suggest that MEF-2 plays a more dominant role in regulating glucose transport in hibernators. As well, the coordinated regulation of glucose transport during hibernation is likely a mechanism of reversible insulin resistance, and suggests the potential importance of MEF-2 in rescuing the GLUT-4 protein deficiency observed in T2DM patients.

\section{PPAR- $\gamma /$ PGC-1 $\alpha$ regulation in T2DM and hibernation}

Excess fat accumulation in adipose tissue has been considered to be one of the main associations between obesity and diabetes (6). One of the key regulators that modulate adipogenesis is PPAR- $\gamma$, a transcription factor that regulates the expression of genes involved in adipose differentiation (35). The activation of PPAR- $\gamma$ initiates the differentiation of insulin-sensitive fat cells that increases insulin-directed glucose uptake (36). PPAR- $\gamma$ achieves this control through the downstream regulation of several genes that are involved in insulin sensing, including tumor necrosis factor- $\alpha$ (TNF- $\alpha$ ) and leptin. This is in addition to genes involved in fatty acid metabolism such as lipoprotein lipase and adipocyte/heart type fatty-acid binding proteins (Figure 2A) (36). Defects in PPAR- $\gamma$ have been observed in T2DM patients, with individuals exhibiting deleterious mutations in the PPAR- $\gamma$ gene showing symptoms of severe insulin resistance, likely contributed by dysfunctional adipose metabolism (Figure 2B) (37).

Another transcription factor that is involved in T2DM is the PCG-1 $\alpha$ (38). The PGC-1 $\alpha$ protein functions mainly as a coactivator that regulates PPAR- $\gamma$ activity as well as other biological functions including mitochondria biogenesis and fatty acid oxidation (39-41). It has been well documented that mitochondrial dysfunction is one of the 

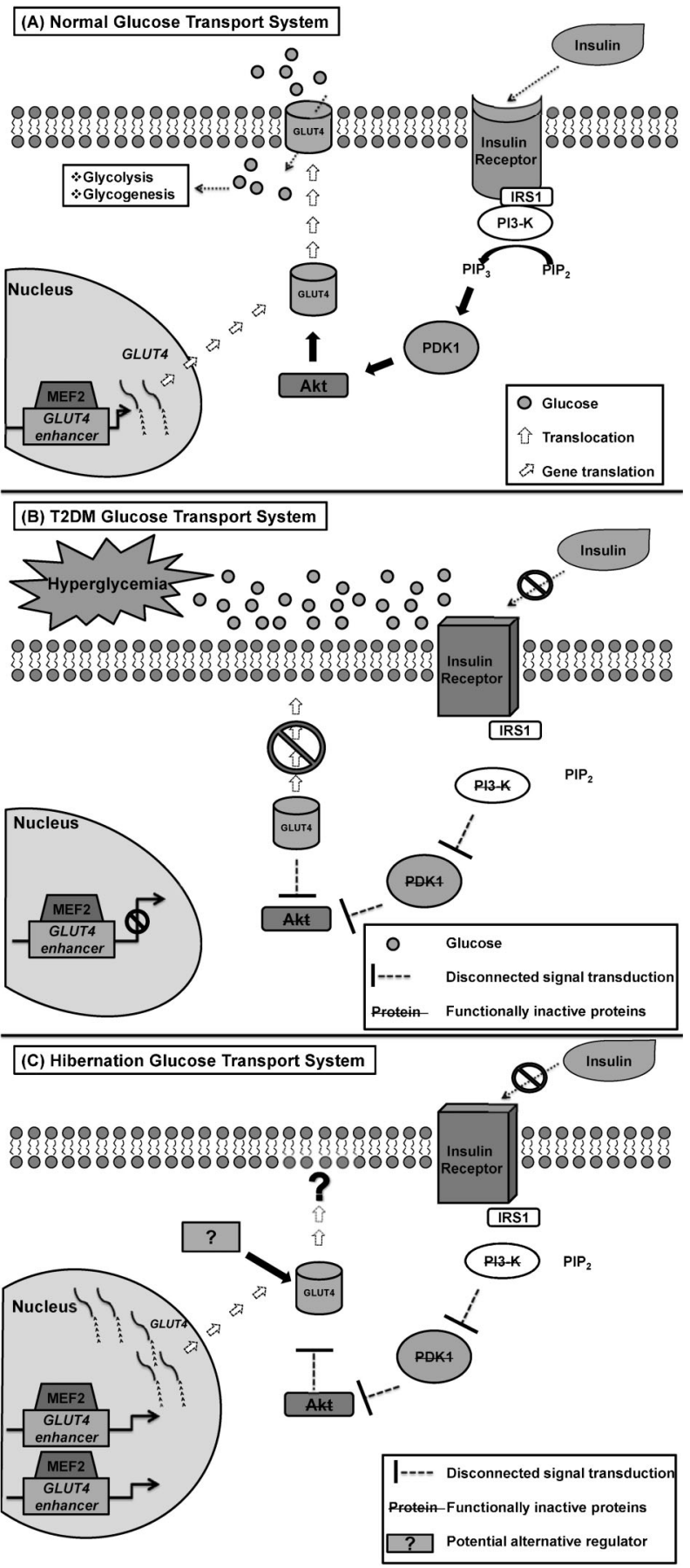

metabolic disorders exhibited by T2DM patients, and it has been hypothesized that these dysfunctions are associated with the dysregulation of PGC-1 $\alpha$ (38). Previous studies found that the expression of PGC-1 $\alpha$ was downregulated in the adipose tissue and skeletal muscle of individuals with T2DM (42). At the molecular level, a downregulation in PGC-1 $\alpha$ expression can lead to
Figure 1. Insulin-signaling pathway. Interactions between insulin molecules and the insulin receptor initiate a signal transduction cascade via the activation of PI3-K. The downstream cascade stimulates the activation of Akt, which facilitates the translocation of GLUT-4 transporter towards the plasma membrane, where active glucose transport takes place. The transcription factor MEF-2 interacts with the GLUT4 enhancer regions to promote the transcription of GLUT4, and upregulate GLUT-4 expression. A, Functional glucose transport system, where transported glucose is either stored via glycogenesis, or broken down via glycolysis. $B$, T2DM glucose transport system, where insulin resistance inhibits the activation of $\mathrm{PI} 3-\mathrm{K}$, and disrupts the signal transduction pathway that facilitates the translocation of GLUT-4 transporter. Accumulation of glucose molecules leads to the onset of hyperglycemia. C, Hibernation glucose transport system. Reversible suppression of carbohydrate-based metabolism during hibernation results in the deliberate downregulation of the Akt signal transduction pathway. However, enhanced MEF-2 activity coupled with upregulated GLUT4 expression during late hibernation suggests the possibility of alternative metabolic pathways that regulate the GLUT-4 transport system.

a decrease in the expression of crucial genes responsible for mitochondrial $\beta$-oxidation of fatty acids (Figure $2 \mathrm{~B}$ ) (38). Indeed, the mitochondrial dysfunctions observed in T2DM individuals consist of a decrease in both oxidative phosphorylation and fatty acid oxidation, which include a reduction in activities of $\mathrm{NADH}-\mathrm{O}_{2}$ oxidoreductase, citrate synthase, and mitochondrial complex I $(5,43)$. The decrease in mitochondrial fatty acid oxidation results in an accumulation of free fatty acids (FFA) derivatives such as fatty acyl CoA, diacylglycerol, and ceramides (44). It has been proposed that this buildup of FFA derivatives can lead to insulin resistance through the inactivation of insulin receptor substrate-1 (IRS-1), a protein responsible for activating the insulin-dependent Akt signaling cascade $(5,44)$.

Due to the primary reliance on lipid catabolism in hibernators, the regulation of PPAR- $\gamma$ and PGC- $1 \alpha$ has been shown to play a major role in the survival of hibernation. In a study by Eddy et al. (45), it was observed that the protein expression of PPAR- $\gamma$ increased significantly in the brown adipose tissue (BAT) of ground squirrels during hibernation. As well, the protein expression of PGC-1 $\alpha$ was upregulated in several tissues including white adipose tissue, BAT, heart, and skeletal muscle (45). The increase in PPAR- $\gamma$ expression was associated with increased gene expression of adipocyte fatty acid binding proteins (A-FABP) and heart-type FABP (H-FABP) (Figure 2C) (46). FABP is a chaperone protein that is responsible for binding and shuttling fatty acids to various cell compartments. Hibernators are unique in that they retain a high level of BAT, a tissue that is packed with mitochondria specialized for non-shivering thermogenesis (47). Due to the important role of BAT during hibernation, the increase in expression of PPAR- $\gamma$ is thought to be associated with an increased translocation of lipids, as metabolic fuel, via A-FABP (46). It is likely that most of the 


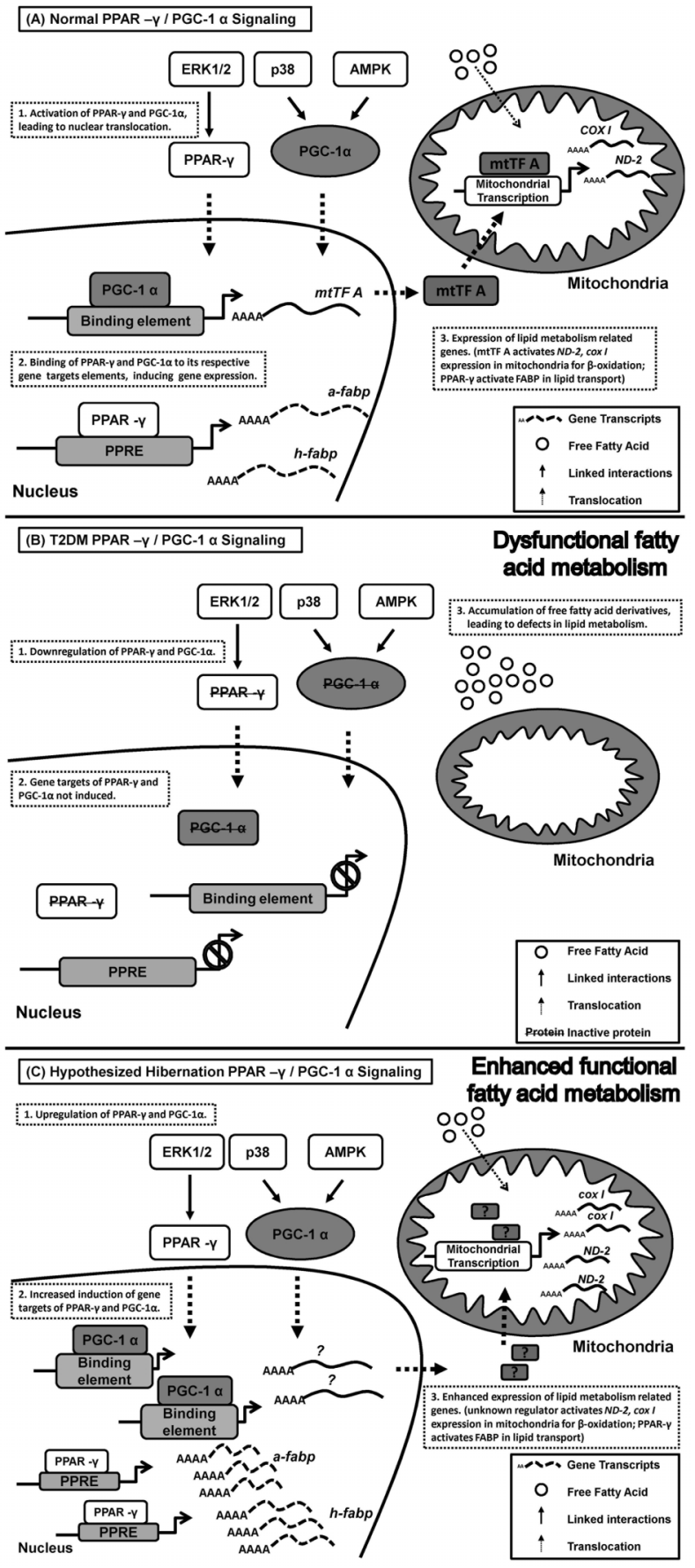

lipid is shuttled to the mitochondria, where it is used mainly to fuel the massive increase in non-shivering thermogenesis in BAT that powers the rewarming of the hibernator's body during arousal from torpor. Meanwhile, the increase in the expression of PGC- $1 \alpha$ during hibernation has been linked with the upregulation of genes involved in the mitochondrial biogenesis including
Figure 2. PPAR- $\gamma /$ PGC- $1 \alpha$ signaling pathways. Activation of PPAR- $\gamma$ through upstream kinase cascades leads to the nuclear translocation of the transcription factor. The binding of PPAR- $\gamma$ onto peroxisome proliferator response elements (PPRE) promotes the expression of genes involved in fatty acid transport such as a-fabp and $h$-fabp. Similar to PPAR- $\gamma$, activation of PGC$1 \alpha$ via upstream kinases facilitates the nuclear translocation of PGC-1 $\alpha$. Upon interacting with various potential binding partners, PGC-1 $\alpha$ stimulates the expression of mitochondrial transcription factor A (mtTFA). mtTFA subsequently translocates into mitochondria and activates expression of $N D-2$ and $\operatorname{cox} I$, genes that are involved in the mitochondrial fatty acid oxidation process. $A$, Normal PPAR- $\gamma /$ PGC- $1 \alpha$ signaling. B, T2DM PPAR- $\gamma /$ PGC- $1 \alpha$ signaling. Downregulation/dysfunctional PPAR- $\gamma$ and PGC- $1 \alpha$ protein expression in T2DM results in the dysregulation of their respective functions. These defects result in the downregulation of PPAR- $\gamma /$ PGC- $1 \alpha$ downstream target genes, leading to the loss of mitochondrial oxidation capacities. The decrease in mitochondrial fatty acid oxidation results in the dysfunction of fatty acid metabolism, leading to the accumulation of free fatty acids. $C$, Hibernation PPAR- $\gamma /$ PGC- $1 \alpha$ signaling. The reversible activation of lipid-based metabolism during hibernation is supported by an enhanced PPAR- $\gamma$ and PGC- $1 \alpha$ protein expression. Through unidentified regulators, the upregulation of PGC- $1 \alpha$ results in the activation of $N D-2$ and $\operatorname{cox} I$, two mitochondrial genes that are critical in the mitochondrial fatty acid $\beta$-oxidation process. The upregulation of PPAR- $\gamma$ during hibernation leads to the promotion of A-FABP and $\mathrm{H}-\mathrm{FABP}$, isoforms of fatty acid-binding proteins that facilitate and enhance lipid transportation during hibernation.

NADPH-ubiquinone oxidoreductase chain $2(N D-2)$ and cytochrome $c$ oxidase I (cox I) (Figure 2C) $(45,48,49)$. Although the exact mechanism for the upregulation of mitochondrial genes is not yet identified in ground squirrels, previous studies have shown that PGC- $1 \alpha$ activates the expression of mitochondrial transcription factor A (mtTFA), which subsequently translocates into the mitochondria and activates mitochondrial-encoded genes such as cox I (Figure 2A) (40). The increase in expression of $N D-2$ and cox I genes during hibernation suggests an enhanced capacity of mitochondrial $\beta$-oxidation of lipids, possibly via an increased mitochondrial count.

Although hibernators experience a similar obesity-like condition as T2DM patients, there is a large difference in the regulation of their respective lipid metabolism. The increase in adipose content observed in the hibernators is associated with an enhanced lipid catabolism. In a cellular system where the majority of the metabolic processes are suppressed, an increase in the expression of genes involved in lipid catabolism indicates that adipose metabolism is highly crucial during hibernation. In contrast to hibernation, the lipid-based metabolism of T2DM has been shown to be subject to multiple defects. The primary complication of adipose dysfunction in T2DM is a decrease in the oxidative capacity of lipid metabolism, which is thought to be caused by a dysregulated expression of PPAR- $\gamma$ and PGC- $1 \alpha$. An overall increase in free fatty acid derivative content has been implicated in 
the development of insulin resistance (37). The differences in the mechanism of adipose regulation between hibernation and T2DM have provided clues to potential therapeutic targets of interest in reversing insulin resistance. In both conditions, PPAR- $\gamma$ and PGC- $1 \alpha$ are key regulators in determining the functionality of adipose metabolism, where changes in the expression of these two regulators can lead to either an increase or a decrease in mitochondrial $\beta$-oxidation of fatty acids. Indeed, the use of PPAR- $\gamma$ agonists such as the thiazolidinediones has been shown to temporarily increase insulin sensitivity in T2DM (50). However, future research is still required to determine the molecular mechanisms that are responsible for the activation of PPAR- $\gamma$ and PGC- $1 \alpha$ expression during hibernation, since a coordinated fatty acid metabolism is likely a component of reversible insulin resistance.

\section{Akt signaling pathway regulation in T2DM and hibernation}

Insulin signaling is initiated with the binding of insulin molecules to receptor ligands on the cell membrane. Ligand binding initiates the autophosphorylation and subsequent activation of the insulin receptors (51). Upon activation, the receptors propagate their biological response by activating IRS via phosphorylation. In total, 12 different IRS isoforms can then interact with their corresponding downstream targets to promote the signal cascade (51). IRS-1, the first to be discovered, contains a binding site for the regulatory subunit of phosphatidylinositol 3-kinase (PI3-K). The docking of the PI3-K regulatory subunit with IRS-1 activates the catalytic site of PI3-K, resulting in the phosphorylation and production of phosphatidylinositol 3,4,5-triphosphate $\left(\mathrm{PIP}_{3}\right)$, a lipid second messenger required for the activation of 3-phosphoinositide-dependent protein kinase-1 (PDK1) (Figure 1A) (52). PDK1 is then responsible for activating Akt, also known as protein kinase $\mathrm{B}$, a serine/threonine kinase that is involved in a diverse number of cellular processes including cellular growth and differentiation, cellular survival, and glucose metabolism (53). Major roles of Akt in carbohydrate metabolism include promoting glucose transport via interaction with GLUT-4 and facilitating glycogenesis through interaction with glycogen synthase 3 kinase- $\beta$ (GSK3- $\beta$ ) (54). A defect in either process can lead to the pathogenesis of T2DM $(54,55)$.

Previous studies in obese mouse models (C57BL/KsJ$L_{\text {epr }}{ }^{\mathrm{db} / \mathrm{db}}$ ) have shown that mice exhibiting both insulin resistance and T2DM showed a significant decrease in their content of phosphorylated Akt ${ }^{\text {Ser473. The decrease in }}$ phospho-Akt ${ }^{\mathrm{Ser} 43}$ indicated a reduction in Akt activation and was coupled with a decrease in Akt activity on GSK3$\beta$ (55). Krook et al. (56) also showed a reduction of Akt kinase activity in the skeletal muscle of T2DM patients compared to healthy individuals upon high doses of insulin stimulation, suggesting that T2DM patients exhibit impaired insulin-stimulated Akt signaling. The decrease in Akt activity observed in T2DM patients would not only decrease the rate of glucose transport into myocytes via GLUT-4, but also disrupt glycogenesis by activating GSK3- $\beta$ (Figure 1B). Hence, Akt is a major contributor to a disruption of glucose homeostasis in the skeletal muscle and has become a primary target of therapeutic interest in T2DM.

The Akt signaling pathway is a well-studied kinase cascade in hibernating ground squirrels, particularly with respect to its potential role in coordinating suppression of protein synthesis. McMullen and Hallenbeck (57) found that the level of phospho-Akt ${ }^{\text {Ser473 }}$ in the liver of $I$. tridecemlineatus decreased significantly by 57 and $77 \%$ during early and late stages of hibernation, respectively. Another study examining skeletal muscle of $I$. tridecemlineatus found that the phospho-Akt ${ }^{\text {Ser473 }}$ content was reduced by $55 \%$ during the late stages of hibernation, followed by an overall suppression of the downstream mTOR signaling network and contributing to a state of translational arrest (58). Enzymatic studies of Akt in hibernating Richardson's ground squirrels (I. richardsonii) have also shown a decrease in Akt kinase activity during torpor in both the skeletal muscle $\left(\mathrm{V}_{\max }\right.$ decrease of $\left.60 \%\right)$ and the liver $\left(\mathrm{V}_{\max }\right.$ decrease of $66 \%$ ) (59). In the same study, a decrease in phospho-Akt ${ }^{\mathrm{Ser} 473}$ content was correlated with a decrease in the enzymatic activity of Akt in both skeletal muscle and liver.

This type of Akt inactivation was also observed in patients with T2DM. A decrease in the Akt kinase activity was observed to be associated with a decrease in GSK3$\beta$ phosphorylation, and was also linked to a decrease in GLUT-4 activation. The experimental conclusions of multiple Akt studies in hibernators would suggest that the glucose homeostasis and glycogenesis might be affected due to the coordinated suppression of the Akt signaling pathway. Interestingly, in the early arousal stages of torpor, the level of phospho-Akt ${ }^{\text {Ser473 }}$ increased significantly by 3.2 -fold (57). As well, during the interbout euthermia between torpor bouts, the levels of phospho$\mathrm{Akt}^{\mathrm{Ser} 473}$ returned to the levels observed in the euthermic control stage. The rapid increase in Akt activity during the arousal from torpor could indicate a re-activation of the insulin signaling pathway and could suggest the reversal of insulin resistance upon the exit of hibernation.

Interestingly, unlike the regulation of glucose and fatty acid metabolism, the pattern of Akt regulation in hibernators appears to be similar to the dysregulation that is often observed in T2DM patients. Despite the findings of reversible Akt activation during the arousal stages of the hibernation cycle, little is known about the mechanism that is responsible for such an event. Although it remains unclear whether the suppression of Akt during hibernation is a result of prolonged insulin resistance or a suppression 


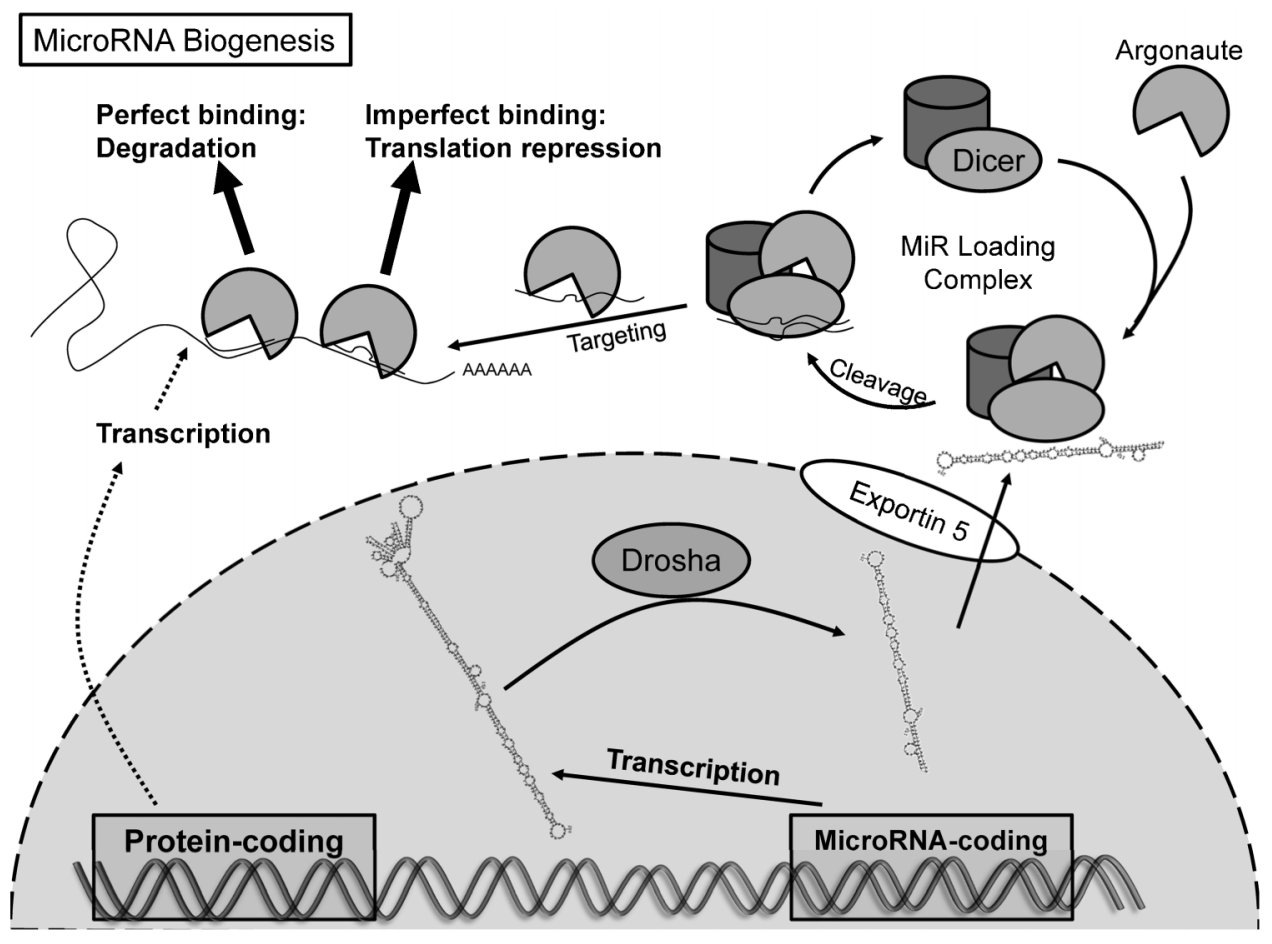

Figure 3. MicroRNA biogenesis pathway. Primary transcripts are transcribed by RNA polymerase II and excised into single-stranded mature microRNA structures. Mature microRNA structures are then loaded into the microRNA-induced silencing complex and influence mRNA expression through degradation (perfect binding) or translational repression (imperfect binding) through 3-untranslated region binding.

of Akt upstream regulators (PI3-K, IRS-1, etc.), the restoration of Akt phosphorylation during arousal from hibernation could suggest a potential mechanism that rescues Akt kinase activity. The restoration of Akt activity could function to recover cellular process such as glucose metabolism that is often impaired through suppressed Akt activity in T2DM patients. Nonetheless, further investigation into the regulation of Akt upstream regulators such as PI3-K, IRS-1, and insulin receptors during hibernation would provide more insights into the state of insulin signaling during torpor, and its effects on the Akt kinase.

\section{Future links between T2DM and hibernation: non-coding RNA}

In the last few decades, few biological disciplines have undergone the growth of small non-coding RNAs, microRNA. These 18-25-nucleotide-long transcripts are able to bind with full or partial complementarity, usually to the 3' untranslated regions of mRNA targets, resulting in the inhibition of translation or degradation of that target (Figure 3) (60-62). Simply due to their sequence diversity, and the fact that their microRNAs are predicted to target the majority of mRNAs in mammals, this regulatory pathway is of great importance. In fact, through a myriad of comparative expression analyses and gain- and lossof-function experiments, microRNAs have been shown to be critically involved in biological development, cell differentiation, apoptosis, cell cycle control, stress response, and disease pathogenesis (60,63-65). Notably, the characteristics of microRNA-induced regulation of gene expression meet the criteria necessary for the mechanisms of metabolic regulation to be broadly applicable, readily coordinated, easily induced, and readily reversed without wholesale reorganization of the cell (66). Although microRNAs are implicated in a variety of disease states, only a handful of studies have demonstrated the involvement of microRNAs in the development of T2DM (67-70). Interestingly, several of the microRNAs found to be differentially expressed in T2DM models are also found to be differentially regulated during metabolic rate depression throughout mammalian hibernation. Although research in this field is only beginning, the extensiveness of gene regulation by non-coding RNA is supported by a finding that only one fifth of transcription across the genome is associated with protein-coding genes, suggesting at least four times more non-coding than coding RNA sequences $(71,72)$. 


\section{MicroRNA: the link between T2DM and hibernation}

Despite the absence of microRNAs from the current lists of genes shown to be associated with the onset and development of T2DM, an involvement of microRNAs seems likely given the evidence provided by available studies (73). One report documented an altered microRNA pattern, contributing to FFA-induced pancreatic $\beta$-cell dysfunction (67). Particularly, it was found that miR-34a is a transcriptional target of p53, a transcription factor capable of inducing apoptosis in several diseases. Additionally, miR-34a is able to induce the degradation of $\beta$-cell lymphoma $2(\mathrm{Bcl} 2) \mathrm{mRNA}$, which encodes an antiapoptotic protein $(74,75)$. Most notably, both p53 and the decreased expression of anti-apoptotic Bcl2 mRNA play critical roles in the pancreas involving the apoptosis of insulin-secreting cells (67). Additionally, within the pancreatic islets, miR-34a has been shown to affect hormone secretion through the targeting and mRNA downregulation of vesicle-associated membrane protein 2 (VAMP2), a vesicle protein that is involved in insulin exocytosis. Therefore, the differential regulation of p53induced miR-34a expression may facilitate some of the negative outcomes induced by FFA in pancreatic function and the survival of $\beta$-cells, mimicking the state of obesityassociated T2DM. Furthermore, microRNA regulation of insulin exocytosis has been expanded with the discovery of differentially expressed miR-375 microRNAs in pancreatic endocrine cell lines (76). However, when overexpressed, this particular microRNA resulted in the suppression of glucose-stimulated insulin release, while inhibition of this microRNA restored normal insulin exocytosis. Additionally, miR-375 has also been reported to target PDK1 in the pancreatic islet cells. PDK1, in the presence of $\mathrm{PIP}_{3}$, stimulates the insulin-responsive $\mathrm{PI} 3-\mathrm{K} /$ Akt pathway and overexpression of miR-375 would diminish this effect, decreasing PI3-K/Akt signaling (77). This result establishes miR-375 as a potentially important modulator of insulin regulation, further implicating the involvement of microRNAs in T2DM.

As mentioned earlier in this review, the phosphorylation state of Akt is a key point of regulation and determines $\mathrm{PI} 3-\mathrm{K} / \mathrm{Akt}$ pathway activity or inactivity (78). Protein phosphatases (primarily phosphatase and tensin homolog, PTEN) act to suppress this signal by removing phosphate groups from members of the pathway (78). Numerous microRNAs have been experimentally shown to act as negative regulators of the $\mathrm{PI} 3-\mathrm{K} / \mathrm{Akt}$ signaling pathway $(79,80)$. Of interest is miR-29, a microRNA shown to negatively affect the activation of the PI3-K/Akt pathway through repression of $\mathrm{PI} 3-\mathrm{K}$ regulatory subunits (80). Importantly, this microRNA was found to be highly elevated in the skeletal muscle of diabetic rats, acting to inhibit the typical insulin response (69). This included a significant repression of insulin-stimulated glucose uptake and the inhibition of insulin-stimulated Akt signaling, most likely mediated through $\mathrm{PI} 3-\mathrm{K}$ regulation. Given the importance of PI3-K/Akt signaling in T2DM, miR-29 has the potential to act as a mechanism to dampen insulin signaling.

Apart from their roles in T2DM, the regulation of distinct microRNAs has been well established as part of the typical stress response. To date, thousands of publications have documented their role in a multitude of cell function and dysfunction. A recent study showed that one aspect of metabolic rate depression may be the differential expression of key microRNA species during torpor (71). The first study to characterize microRNAs in hibernation examined the expression of nine microRNA species in tissues from euthermic versus hibernating ground squirrels; several of these were identified to be differentially expressed in kidney, skeletal muscle and heart along with elevated amounts of the microRNA processing enzyme, Dicer, during torpor (81). Although these do not address all differentially expressed microRNAs in hibernators, these experiments begin to examine the critical roles that non-coding RNAs may play during hibernation, and consequently may facilitate reversible insulin resistance. As previously mentioned, two key microRNAs have been identified to be differentially regulated in both hibernation and T2DM, namely, miR-34a and miR-29. miR-34a has been found to increase approximately 2 -fold during late torpor in liver from hibernating arctic ground squirrels (I. parryii) (82). The increase of miR-34a in the liver of a mammalian hibernator may suggest a regulatory role in apoptosis contributing to glucose secretion. Additionally, preliminary RT-PCR studies, using a modified stem-loop microRNA amplification protocol, conducted in the skeletal muscle of hibernating little brown bats (Myotis lucifugus) have identified an increase of $1.3 \pm 0.1$-fold in mature miR29 during late torpor $(83,84)$. An increase of miR-29 in skeletal muscle may be indicative of insulin-stimulated glucose uptake regulating the inactivation of the PI3-K/Akt signaling pathway similar to insulin resistant models. These studies suggest a similar regulation of both the PI3-K/Akt and p53 pathways in hibernators and T2DM, which may provide an indication of the important contributing role of microRNA expression patterns in reversible insulin resistance.

Identification of similar microRNA expression patterns between models of reversible and irreversible insulin resistance may identify a mechanism of reversing T2DM, accomplished through the decreased expression of key microRNAs including, perhaps, miR-34a and miR-29. The complexity of the transcriptome, and our evolving understanding of its structure, may inform a re-interpretation of the functional basis for many microRNAs associated with the diabetic state and a comparative approach to hibernating mammals may aid in this discovery. 


\section{Conclusion}

This review attempts to summarize the current state of T2DM research, and draws parallels to the molecular pathways that may contribute to the reversible insulin resistance in mammalian hibernation. We have considered the relevance of hibernation in ground squirrels to human physiology and insulin resistance. Hibernators are a remarkable model for human obesity-like pathologies, fuelling the torpor cycle with adipocyte hypertrophy, hyperphagia, and reversible insulin resistance. Most notable is the marked insulin resistance during the period of fat storage, followed by its reversal as animals enter hibernation. This mode of action is likely facilitated through post-translational modifications to the key signaling transduction pathways mentioned in this review, with similar pathways commonly seen to be dysregulated in T2DM. The difference, however, lies within the ability of ground squirrels to actively regulate these signaling pathways, in contrast to the dysregulation observed in T2DM patients. Furthermore, the abundance of signal transducer proteins in a network can influence both the direction and strength of signaling and may play a role in remodeling the signaling landscape in the insulinresistant hibernator. Since microRNAs are known to play

\section{References}

1. Cummings BP, Strader AD, Stanhope KL, Graham JL, Lee $\mathrm{J}$, Raybould HE, et al. lleal interposition surgery improves glucose and lipid metabolism and delays diabetes onset in the UCD-T2DM rat. Gastroenterology 2010; 138: 24372446, doi: 10.1053/j.gastro.2010.03.005.

2. Kadowaki T. Insights into insulin resistance and type 2 diabetes from knockout mouse models. J Clin Invest 2000; 106: 459-465, doi: $10.1172 / \mathrm{JCl} 10830$.

3. Jin W, Patti ME. Genetic determinants and molecular pathways in the pathogenesis of type 2 diabetes. Clin Sci 2009; 116: 99-111, doi: 10.1042/CS20080090.

4. Camps M, Castello A, Munoz P, Monfar M, Testar X, Palacin M, et al. Effect of diabetes and fasting on GLUT-4 (muscle/fat) glucose-transporter expression in insulinsensitive tissues. Heterogeneous response in heart, red and white muscle. Biochem J 1992; 282 (Part 3): 765-772.

5. Lowell BB, Shulman GI. Mitochondrial dysfunction and type 2 diabetes. Science 2005; 307: 384-387, doi: 10.1126/ science. 1104343.

6. Wang MY, Grayburn P, Chen S, Ravazzola M, Orci L, Unger RH. Adipogenic capacity and the susceptibility to type 2 diabetes and metabolic syndrome. Proc Natl Acad Sci U S A 2008; 105: 6139-6144, doi: 10.1073/ pnas. 0801981105 .

7. Martin SL. Mammalian hibernation: a naturally reversible model for insulin resistance in man? Diab Vasc Dis Res 2008; 5: 76-81, doi: 10.3132/dvdr.2008.013.

8. Dark J. Annual lipid cycles in hibernators: integration of physiology and behavior. Annu Rev Nutr 2005; 25: 469-497, doi: 10.1146/annurev.nutr.25.050304.092514. roles in altering protein expression, it has been proposed that microRNAs could play crucial roles in the regulation of cellular signaling (85). However, many key areas have not yet been evaluated in hypometabolic systems. The purpose of developing insulin resistance in hibernators, for example, has not been fully explored and may play a role in energy storage or in maintaining glucose stores for brain utilization, while other tissues become insulin resistant. Although this review presents many critical targets likely to contribute to the reversible insulin resistance seen in hibernators, future study will have to focus on the degree of insulin resistance found during different stages of hibernation, validation of the critical pathways contributing to reversible insulin resistance, and the elucidation of microRNAs as key regulators.

\section{Acknowledgments}

Thanks are due to J.M. Storey and H.A. Holden for editorial review of the manuscript. Research in the K.B. Storey laboratory is supported by a Discovery grant from NSERC. K.B. Storey holds the Canada Research Chair in Molecular Physiology, C.-W. Wu and K.K. Biggar hold NSERC postgraduate fellowships. Research supported by NSERC Canada (Grant \#6793).

9. Storey KB. Out cold: biochemical regulation of mammalian hibernation - a mini-review. Gerontology 2010; 56: 220-230, doi: $10.1159 / 000228829$.

10. Wang LCH, Lee TF. Torpor and hibernation in mammals: metabolic, physiological and biochemical adaptations. New York: Oxford University Press; 1996.

11. Guppy M, Withers P. Metabolic depression in animals: physiological perspectives and biochemical generalizations. Biol Rev Camb Philos Soc 1999; 74: 1-40, doi: 10.1017/ S0006323198005258.

12. Storey KB, Storey JM. Tribute to P.L. Lutz: putting life on 'pause' - molecular regulation of hypometabolism. J Exp Biol 2007; 210: 1700-1714, doi: 10.1242/jeb.02716.

13. Shepherd PR, Kahn BB. Glucose transporters and insulin action - implications for insulin resistance and diabetes mellitus. N Engl J Med 1999; 341: 248-257, doi: 10.1056/ NEJM199907223410406.

14. Bouche C, Serdy S, Kahn CR, Goldfine AB. The cellular fate of glucose and its relevance in type 2 diabetes. Endocr Rev 2004; 25: 807-830, doi: 10.1210/er.2003-0026.

15. Balasubramanyam M, Mohan V. Current concepts of PPARgamma signaling in diabetes mellitus. Current Science 2000; 79: 1440-1446.

16. Kerouz NJ, Horsch D, Pons S, Kahn CR. Differential regulation of insulin receptor substrates-1 and -2 (IRS-1 and IRS-2) and phosphatidylinositol 3-kinase isoforms in liver and muscle of the obese diabetic (ob/ob) mouse. J Clin Invest 1997; 100: 3164-3172, doi: 10.1172/JCI119872.

17. Buck MJ, Squire TL, Andrews MT. Coordinate expression of the PDK4 gene: a means of regulating fuel selection in a 
hibernating mammal. Physiol Genomics 2002; 8: 5-13.

18. Florant GL, Lawrence AK, Williams K, Bauman WA. Seasonal changes in pancreatic B-cell function in euthermic yellow-bellied marmots. Am J Physiol 1985; 249: R159R165.

19. Castex C, Tahri A, Hoo-Paris R, Sutter BC. Glucose oxidation by adipose tissue of the edible dormouse (Glis glis) during hibernation and arousal: effect of insulin. Comp Biochem Physiol A Comp Physiol 1987; 88: 33-36, doi: 10.1016/0300-9629(87)90094-6.

20. Hoo-Paris R, Castex C, Sutter BC. Plasma glucose and insulin in the hibernating hedgehog. Diabete Metab 1978; 4: 13-18.

21. McCarley $\mathrm{H}$. Annual cycle population dynamics and adaptive behaviors of Citellus tridecemlineatus. J Mammal 1966; 47: 294-316, doi: 10.2307/1378126.

22. Lowell BB, Spiegelman BM. Towards a molecular understanding of adaptive thermogenesis. Nature 2000; 404: 652660.

23. Storey KB. Metabolic regulation in mammalian hibernation: enzyme and protein adaptations. Comp Biochem Physiol A Physiol 1997; 118: 1115-1124, doi: 10.1016/S03009629(97)00238-7.

24. Wu CW, Storey KB. Pattern of cellular quiescence over the hibernation cycle in liver of thirteen-lined ground squirrels. Cell Cycle 2012; 11: 1714-1726, doi: 10.4161/cc.19799.

25. MacDonald JA, Storey KB. Regulation of ground squirrel $\mathrm{Na}^{+} \mathrm{K}^{+}$-ATPase activity by reversible phosphorylation during hibernation. Biochem Biophys Res Commun 1999; 254: 424-429, doi: 10.1006/bbrc.1998.9960.

26. Ormseth OA, Nicolson M, Pelleymounter MA, Boyer BB. Leptin inhibits prehibernation hyperphagia and reduces body weight in arctic ground squirrels. Am J Physiol 1996; 271: R1775-R1779.

27. Shoelson SE, Lee J, Goldfine AB. Inflammation and insulin resistance. J Clin Invest 2006; 116: 1793-1801, doi: 10.1172/JCl29069.

28. DeFronzo RA, Bonadonna RC, Ferrannini E. Pathogenesis of NIDDM. A balanced overview. Diabetes Care 1992; 15: 318-368, doi: 10.2337/diacare.15.3.318.

29. Napoli R, Hirshman MF, Horton ES. Mechanisms and time course of impaired skeletal muscle glucose transport activity in streptozocin diabetic rats. J Clin Invest 1995; 96: 427437, doi: $10.1172 / \mathrm{JCl} 118053$.

30. Abdul-Ghani MA, DeFronzo RA. Pathogenesis of insulin resistance in skeletal muscle. J Biomed Biotechnol 2010; 2010: 476279.

31. Kennedy JW, Hirshman MF, Gervino EV, Ocel JV, Forse RA, Hoenig SJ, et al. Acute exercise induces GLUT4 translocation in skeletal muscle of normal human subjects and subjects with type 2 diabetes. Diabetes 1999; 48: 11921197, doi: 10.2337/diabetes.48.5.1192.

32. Tessier SN, Storey KB. Expression of myocyte enhancer factor-2 and downstream genes in ground squirrel skeletal muscle during hibernation. Mol Cell Biochem 2010; 344: 151-162, doi: 10.1007/s11010-010-0538-y.

33. Black BL, Olson EN. Transcriptional control of muscle development by myocyte enhancer factor-2 (MEF2) proteins. Annu Rev Cell Dev Biol 1998; 14: 167-196, doi: 10.1146/annurev.cellbio.14.1.167.

34. Thai MV, Guruswamy S, Cao KT, Pessin JE, Olson AL.
Myocyte enhancer factor 2 (MEF2)-binding site is required for GLUT4 gene expression in transgenic mice. Regulation of MEF2 DNA binding activity in insulin-deficient diabetes. J Biol Chem 1998; 273: 14285-14292, doi: 10.1074/ jbc.273.23.14285.

35. Matsusue K, Peters JM, Gonzalez FJ. PPARbeta/delta potentiates PPARgamma-stimulated adipocyte differentiation. FASEB J 2004; 18: 1477-1479.

36. Spiegelman BM. PPAR-gamma: adipogenic regulator and thiazolidinedione receptor. Diabetes 1998; 47: 507-514, doi: 10.2337/diabetes.47.4.507.

37. Barroso I, Gurnell M, Crowley VE, Agostini M, Schwabe JW, Soos MA, et al. Dominant negative mutations in human PPARgamma associated with severe insulin resistance, diabetes mellitus and hypertension. Nature 1999; 402: 880883.

38. Mootha VK, Lindgren CM, Eriksson KF, Subramanian A, Sihag S, Lehar J, et al. PGC-1alpha-responsive genes involved in oxidative phosphorylation are coordinately downregulated in human diabetes. Nat Genet 2003; 34 267-273, doi: 10.1038/ng1180.

39. Guan HP, Ishizuka T, Chui PC, Lehrke M, Lazar MA. Corepressors selectively control the transcriptional activity of PPARgamma in adipocytes. Genes Dev 2005; 19: 453461, doi: $10.1101 /$ gad.1263305.

40. Wu Z, Puigserver P, Andersson U, Zhang C, Adelmant G, Mootha $\mathrm{V}$, et al. Mechanisms controlling mitochondrial biogenesis and respiration through the thermogenic coactivator PGC-1. Cell 1999; 98: 115-124, doi: 10.1016/S00928674(00)80611-X.

41. Puigserver $P, W u ~ Z$, Park $C W$, Graves $R$, Wright $M$, Spiegelman BM. A cold-inducible coactivator of nuclear receptors linked to adaptive thermogenesis. Cell 1998; 92: 829-839, doi: 10.1016/S0092-8674(00)81410-5.

42. Semple RK, Crowley VC, Sewter CP, Laudes M, Christodoulides C, Considine RV, et al. Expression of the thermogenic nuclear hormone receptor coactivator PGC1alpha is reduced in the adipose tissue of morbidly obese subjects. Int J Obes Relat Metab Disord 2004; 28: 176-179, doi: 10.1038/sj.ijo.0802482.

43. Kelley DE, He J, Menshikova EV, Ritov VB. Dysfunction of mitochondria in human skeletal muscle in type 2 diabetes. Diabetes 2002; 51: 2944-2950, doi: 10.2337/diabetes.51.10.2944

44. Boden $\mathrm{G}$, Shulman $\mathrm{GI}$. Free fatty acids in obesity and type 2 diabetes: defining their role in the development of insulin resistance and beta-cell dysfunction. Eur J Clin Invest 2002; 32 (Suppl 3): 14-23, doi: 10.1046/j.1365-2362.32.s3.3.x.

45. Eddy SF, Morin P Jr, Storey KB. Cloning and expression of PPAR-gamma and PGC-1alpha from the hibernating ground squirrel, Spermophilus tridecemlineatus. Mol Cell Biochem 2005; 269: 175-182, doi: 10.1007/s11010-0053459-4.

46. Hittel D, Storey KB. Differential expression of adipose- and heart-type fatty acid binding proteins in hibernating ground squirrels. Biochim Biophys Acta 2001; 1522: 238-243, doi: 10.1016/S0167-4781(01)00338-4.

47. Cannon B, Nedergaard J. Brown adipose tissue: function and physiological significance. Physiol Rev 2004; 84: 277359, doi: 10.1152/physrev.00015.2003.

48. Hittel DS, Storey KB. Differential expression of mitochondria- 
encoded genes in a hibernating mammal. J Exp Biol 2002; 205: 1625-1631.

49. Fahlman A, Storey JM, Storey KB. Gene up-regulation in heart during mammalian hibernation. Cryobiology 2000; 40: 332-342, doi: 10.1006/cryo.2000.2254.

50. Smith U, Gogg S, Johansson A, Olausson T, Rotter V, Svalstedt B. Thiazolidinediones (PPARgamma agonists) but not PPARalpha agonists increase IRS-2 gene expression in 3T3-L1 and human adipocytes. FASEB J 2001; 15: 215220, doi: 10.1096/fj.00-0020com.

51. Bjornholm M, Zierath JR. Insulin signal transduction in human skeletal muscle: identifying the defects in Type II diabetes. Biochem Soc Trans 2005; 33: 354-357, doi: 10.1042/BST0330354.

52. De Camilli P, Emr SD, McPherson PS, Novick P. Phosphoinositides as regulators in membrane traffic. Science 1996; 271: 1533-1539, doi: 10.1126/science.271.5255.1533.

53. Zdychova J, Komers R. Emerging role of Akt kinase/protein kinase $\mathrm{B}$ signaling in pathophysiology of diabetes and its complications. Physiol Res 2005; 54: 1-16.

54. Wang Q, Somwar R, Bilan PJ, Liu Z, Jin J, Woodgett JR, et al. Protein kinase B/Akt participates in GLUT4 translocation by insulin in L6 myoblasts. Mol Cell Biol 1999; 19: 40084018.

55. Shao J, Yamashita H, Qiao L, Friedman JE. Decreased Akt kinase activity and insulin resistance in C57BL/KsJ-Leprdb/ db mice. J Endocrinol 2000; 167: 107-115, doi: 10.1677/ joe.0.1670107.

56. Krook A, Roth RA, Jiang XJ, Zierath JR, Wallberghenriksson $\mathrm{H}$. Insulin-stimulated Akt kinase activity is reduced in skeletal muscle from NIDDM subjects. Diabetes 1998; 47: 1281-1286, doi: 10.2337/diabetes. 47.8.1281.

57. McMullen DC, Hallenbeck JM. Regulation of Akt during torpor in the hibernating ground squirrel, Ictidomys tridecemlineatus. J Comp Physiol B 2010; 180: 927-934, doi: 10.1007/s00360-010-0468-8.

58. Wu CW, Storey KB. Regulation of the mTOR signaling network in hibernating thirteen-lined ground squirrels. $J$ Exp Biol 2012; 215: 1720-1727, doi: 10.1242/jeb.066225.

59. Abnous K, Dieni CA, Storey KB. Regulation of Akt during hibernation in Richardson's ground squirrels. Biochim Biophys Acta 2008; 1780: 185-193, doi: 10.1016/j.bbagen. 2007.10.009.

60. Leung AK, Sharp PA. MicroRNA functions in stress responses. Mol Cell 2010; 40: 205-215, doi: 10.1016/ j.molcel.2010.09.027.

61. Grimson A, Farh KK, Johnston WK, Garrett-Engele P, Lim LP, Bartel DP. MicroRNA targeting specificity in mammals: determinants beyond seed pairing. Mol Cell 2007; 27: 91105, doi: 10.1016/j.molcel.2007.06.017.

62. Schier AF, Giraldez AJ. MicroRNA function and mechanism: insights from zebra fish. Cold Spring Harb Symp Quant Biol 2006; 71: 195-203, doi: 10.1101/sqb.2006.71.055.

63. Shi $Y$, Jin $Y$. MicroRNA in cell differentiation and development. Sci China C Life Sci 2010; 52: 205-211.

64. Chan JA, Krichevsky AM, Kosik KS. MicroRNA-21 is an antiapoptotic factor in human glioblastoma cells. Cancer Res 2005; 65: 6029-6033, doi: 10.1158/0008-5472.CAN-050137.

65. Ivey KN, Srivastava D. MicroRNAs as regulators of differentiation and cell fate decisions. Cell Stem Cell 2010 7: 36-41, doi: 10.1016/j.stem.2010.06.012.

66. Biggar KK, Storey KB. The emerging roles of microRNAs in the molecular responses of metabolic rate depression. $J \mathrm{Mol}$ Cell Biol 2011; 3: 167-175, doi: 10.1093/jmcb/mjq045.

67. Lovis P, Roggli E, Laybutt DR, Gattesco S, Yang JY, Widmann $\mathrm{C}$, et al. Alterations in microRNA expression contribute to fatty acid-induced pancreatic beta-cell dysfunction. Diabetes 2008; 57: 2728-2736, doi: 10.2337/db071252.

68. Kim WH, Lee JW, Gao B, Jung MH. Synergistic activation of JNK/SAPK induced by TNF-alpha and IFN-gamma: apoptosis of pancreatic beta-cells via the p53 and ROS pathway. Cell Signal 2005; 17: 1516-1532, doi: 10.1016/j.cellsig. 2005.03.020

69. Poy MN, Spranger M, Stoffel M. microRNAs and the regulation of glucose and lipid metabolism. Diabetes Obes Metab 2007; 9 (Suppl 2): 67-73, doi: 10.1111/j.14631326.2007.00775.x.

70. He A, Zhu L, Gupta N, Chang Y, Fang F. Overexpression of micro ribonucleic acid 29 , highly up-regulated in diabetic rats, leads to insulin resistance in 3T3-L1 adipocytes. Mol Endocrinol 2007; 21: 2785-2794, doi: 10.1210/me.20070167.

71. Kapranov P, Cheng J, Dike S, Nix DA, Duttagupta R, Willingham AT, et al. RNA maps reveal new RNA classes and a possible function for pervasive transcription. Science 2007; 316: 1484-1488, doi: 10.1126/science.1138341.

72. Cheng J, Kapranov P, Drenkow J, Dike S, Brubaker S, Patel $\mathrm{S}$, et al. Transcriptional maps of 10 human chromosomes at 5-nucleotide resolution. Science 2005; 308: 1149-1154, doi: 10.1126/science.1108625.

73. Walker MD. Role of MicroRNA in pancreatic beta-cells: where more is less. Diabetes 2008; 57: 2567-2568, doi $10.2337 / \mathrm{db} 08-0934$

74. Vousden KH, Lane DP. p53 in health and disease. Nat Rev Mol Cell Biol 2007; 8: 275-283, doi: 10.1038/nrm2147.

75. Bommer GT, Gerin I, Feng Y, Kaczorowski AJ, Kuick R, Love RE, et al. p53-mediated activation of miRNA34 candidate tumor-suppressor genes. Curr Biol 2007; 17: 1298-1307, doi: 10.1016/j.cub.2007.06.068.

76. Poy MN, Eliasson L, Krutzfeldt J, Kuwajima S, Ma X, Macdonald $\mathrm{PE}$, et al. A pancreatic islet-specific microRNA regulates insulin secretion. Nature 2004; 432: 226-230, doi: 10.1038/nature03076.

77. El Ouaamari A, Baroukh N, Martens GA, Lebrun $P$, Pipeleers D, van Obberghen E. miR-375 targets $3^{\prime}-$ phosphoinositide-dependent protein kinase-1 and regulates glucose-induced biological responses in pancreatic beta-cells. Diabetes 2008; 57: 2708-2717, doi: 10.2337/db07-1614.

78. Manning BD, Cantley LC. AKT/PKB signaling: navigating downstream. Cell 2007; 129: 1261-1274, doi: 10.1016/ j.cell.2007.06.009.

79. Yamanaka Y, Tagawa H, Takahashi N, Watanabe A, Guo $\mathrm{YM}$, Iwamoto $\mathrm{K}$, et al. Aberrant overexpression of microRNAs activate AKT signaling via down-regulation of tumor suppressors in natural killer-cell lymphoma/leukemia. Blood 2009; 114: 3265-3275, doi: 10.1182/blood-2009-06222794.

80. Small EM, O'Rourke JR, Moresi V, Sutherland LB, McAnally J, Gerard RD, et al. Regulation of PI3-kinase/Akt signaling 
by muscle-enriched microRNA-486. Proc Natl Acad Sci U S A 2010; 107: 4218-4223, doi: 10.1073/pnas.1000300107.

81. Morin P Jr, Dubuc A, Storey KB. Differential expression of microRNA species in organs of hibernating ground squirrels: a role in translational suppression during torpor. Biochim Biophys Acta 2008; 1779: 628-633, doi: 10.1016/ j.bbagrm.2008.07.011.

82. Liu Y, Hu W, Wang H, Lu M, Shao C, Menzel C, et al. Genomic analysis of miRNAs in an extreme mammalian hibernator, the Arctic ground squirrel. Physiol Genomics 2010; 42A: 39-51, doi: 10.1152/physiolgenomics.00054. 2010.

83. Kornfeld SF, Biggar KK, Storey KB. Differential expression of mature microRNAs involved in muscle maintenance of hibernating little brown bats, Myotis lucifugus: A model of muscle atrophy resistance. Genomics Proteomics Bioinformatics 2012 (in press), doi: 10.1016/j.gpb.2012. 09.001.

84. Biggar KK, Kornfeld SF, Storey KB. Amplification and sequencing of mature microRNAs in uncharacterized animal models using stem-loop reverse transcription-polymerase chain reaction. Anal Biochem 2011; 416: 231-233, doi: 10.1016/j.ab.2011.05.015.

85. Cui Q, Yu Z, Purisima EO, Wang E. Principles of microRNA regulation of a human cellular signaling network. Mol Syst Biol 2006; 2: 46, doi: 10.1038/msb4100089. 